\title{
Modelos de regressão aleatória com diferentes estruturas de variância residual para descrever o tamanho da leitegada ${ }^{1}$
}

\author{
Random regression models with different residual variance structures for describing \\ litter size in swine
}

\author{
Aderbal Cavalcante-Neto ${ }^{2}$, Patrícia Tholon ${ }^{3}$, Jeffrey Frederico Lui ${ }^{4}$, Maria Aparecida Cassiano Lara ${ }^{5}$, Carlos \\ Fonseca $^{6}$, Maria Norma Ribeiro ${ }^{7}$ e José Lindenberg Rocha Sarmento ${ }^{8 *}$
}

\begin{abstract}
Resumo - Objetivou-se comparar modelos de regressão aleatória com diferentes estruturas de variância residual, a fim de se buscar a melhor modelagem para a característica tamanho da leitegada ao nascer (TLN). Utilizaram-se 1.701 registros de TLN, que foram analisados por meio de modelo animal, unicaracterística, de regressão aleatória. As regressões fixa e aleatórias foram representadas por funções contínuas sobre a ordem de parto, ajustadas por polinômios ortogonais de Legendre de ordem 3. Para averiguar a melhor modelagem para a variância residual, considerou-se a heterogeneidade de variância por meio de 1 a 7 classes de variância residual. O modelo geral de análise incluiu grupo de contemporâneo como efeito fixo; os coeficientes de regressão fixa para modelar a trajetória média da população; os coeficientes de regressão aleatória do efeito genético aditivo-direto, do comumde-leitegada e do de ambiente permanente de animal; e o efeito aleatório residual. O teste da razão de verossimilhança, o critério de informação de Akaike e o critério de informação bayesiano de Schwarz apontaram o modelo que considerou homogeneidade de variância como o que proporcionou melhor ajuste aos dados utilizados. As herdabilidades obtidas foram próximas a zero $(0,002$ a $0,006)$. O efeito de ambiente permanente foi crescente da $1^{\mathrm{a}}(0,06)$ à $5^{\mathrm{a}}(0,28)$ ordem, mas decrescente desse ponto até a $7^{\mathrm{a}}$ ordem $(0,18)$. O comum-de-leitegada apresentou valores baixos $(0,01$ a 0,02$)$. A utilização de homogeneidade de variância residual foi mais adequada para modelar as variâncias associadas à característica tamanho da leitegada ao nascer nesse conjunto de dado.
\end{abstract}

Palavras-chave - Função de covariância. Parâmetro genético. Fêmea suína.

Abstract - The objective of this work was to compare random regression models with different residual variance structures, so as to obtain the best modeling for the trait litter size at birth (LSB) in swine. One thousand, seven hundred and one records of LSB were analyzed. LSB was analyzed by means of a random-regression, single-characteristic animal model. The fixed and random regressions were represented by continuous functions over the farrowing order, adjusted by third-order Legendre's orthogonal polynomials. To obtain the best modeling for the residual variance, variance heterogeneity was assumed by means of 1 to 7 classes of residual variance. The general analysis model included a contemporary group; the fixed regression coefficients for modeling the population's average trajectory; the random regression coefficients of the direct additive genetic effects both of the litter and of the animal's permanent environment; and the residual random effect. The likelihood-ratio test, Akaike's information criterion, and Schwarz's Bayesian information criterion appointed the model that considered variance homogeneity as being the one that provided the best adjustment to the data used. Overall, the heritabilities obtained were close to zero (0.002 to 0.006). Regarding the permanent environment proportion, different magnitudes were observed for the farrowing order: increasing from the $1^{\text {st }}(0.06)$ to the $5^{\text {th }}(0.28)$ orders and decreasing from there to the $7^{\text {th }}$ order $(0.18)$. The common litter effect presented low values (from 0.01 to 0.02 ). The use of residual variance homogeneity was more suitable for modeling variances associated to the trait litter size at birth in this data set.

Key words - Covariance functions. Genetic parameter. Sow.

\footnotetext{
* Autor para correspondência

${ }^{1}$ Recebido para publicação em 14/09/2010; aprovado em 14/03/2011 programa de bolsas de alto nível para a América Latina.

2Departamento de Biologia/CESAM, Universidade de Aveiro, Portugal, aderbalcavalcante@gmail.com

${ }^{3}$ Embrapa Pecuária Sudeste, São Carlos, Brasil, ptholon@cppse.embrapa.br

${ }^{4}$ Departamento de Zootecnia, Campus de Jaboticabal/UNESP, Brasil, jeffrey@fcav.unesp.br

${ }^{5}$ Instituto de Zootecnia, Nova Odessa, São Paulo-SP, Brasil, malara@iz.sp.gov.br

${ }^{6}$ Departamento de Biologia/CESAM, Universidade de Aveiro, Portugal, cfonseca@bio.ua.pt

${ }^{7}$ Departamento de Zootecnia/UFRPE, Recife-PE, Brasil, ribeiromn1@hotmail.com

${ }^{8}$ Campus de Bom Jesus da Universidade Federal do Piaui, Brasil, sarmento@ufpi.edu.br
}

Trabalho realizado com o apoio financeiro da FCT - Fundação para a Ciência e Tecnologia (SFRH/BD/47821/2008)e do ALBAN (nº E07D402597BR), 


\section{Introdução}

O tamanho da leitegada ao nascer pode ser analisado sob diferentes modelos, sendo mais simples e, até o momento, mais usual analisá-lo pelo de repetibilidade, assumindo-se que as leitegadas oriundas das diferentes ordens de parto são medidas repetidas da característica (CHEN et al., 2003; KAUFMANN et al., 2000). Entretanto esse modelo pressupõe que a correlação entre as medidas repetidas é igual à unidade, portanto todas as (co) variâncias genéticas e fenotípicas entre as diferentes medidas são de mesma magnitude, ou seja, os mesmos genes controlam o tamanho da leitegada no decorrer das parições, o que é uma modelagem simplista.

Características longitudinais, como o tamanho da leitegada ao nascer, também podem ser analisadas por meio de modelos animais multicaracterísticas (HANENBERG et al., 2001; IRGANG et al., 1994). Nesses modelos, assume-se que cada ordem de parição é uma característica diferente. As correlações entre as médias nas diferentes parições podem ser diferentes da unidade, ou seja, as correlações são levadas em consideração na análise, embora não se faça qualquer pressuposição sobre a estrutura de covariância.

Os modelos de regressão aleatória (MRA) têm sido amplamente reconhecidos em análises de dados longitudinais no melhoramento animal e utilizados para modelar várias características em algumas espécies de animais domésticos, como suínos (HUISMAN et al., 2002; LUKOVIC et al., 2003; LUKOVIC et al., 2004), ovinos (SARMENTO et al., 2006), bovinos de corte (NOBRE et al., 2003) e de leite (VAN DER WERF et al., 1998), entre outros. Os MRA surgiram como uma alternativa capaz de modelar, adequadamente, características longitudinais no contexto do melhoramento animal, a exemplo do tamanho da leitegada ao nascer. Ajustando-se um modelo de regressão aleatória, assume-se, implicitamente, uma determinada estrutura de covariância entre as ordens de parto, que é imposta pelo MRA escolhido. Assim, consideram-se as correlações na análise e assume-se uma determinada estrutura para as correlações.

Nos MRA, a modelagem que considera as variâncias residuais heterogêneas pode melhorar a partição da variação total, mas proporciona um aumento no número de parâmetro a ser estimado no processo de maximização da função de verossimilhança. Segundo El Faro et al. (2008), o número elevado de parâmetro no modelo, a estrutura de covariância e o fato de as matrizes de incidência serem mais densas que os modelos convencionais podem dificultar a convergência na estimação dos componentes de variância, sendo entraves na utilização dessa metodologia para avaliações genéticas com grande volume de dado.
Este trabalho tem por objetivo comparar modelos de regressão aleatória com diferentes estruturas de variância residual, a fim de se buscar a melhor modelagem para as variâncias associadas à característica tamanho da leitegada ao nascer.

\section{Material e métodos}

Analisaram-se 1.701 registros de tamanho da leitegada ao nascer (TLN) - nascidos morto e vivo - da $1^{\text {a à }} 7^{\mathrm{a}}$ ordem de parição de fêmeas Dalland (C-40), pertencentes à Granja São José-Irmãos Canalli (rebanho 1), situada no município de Monte Alto, SP, e à Suinocultura São José (rebanho 2), situada no município de Cabo Verde, MG. Os animais estavam alojados em instalações convencionais de alvenaria de tijolo, revestidas de cimento e de piso impermeável, com piquetes nas unidades destinadas à reprodução. A ração, na forma farelada, era formulada à base de milho e farelo de soja, suplementada com minerais e vitaminas, atendendo às exigências nutricionais de todas as fases da criação; os animais eram vermifugados sistematicamente.

Foram consideradas, para análise, apenas informações oriundas de porcas filhas de reprodutores com, no mínimo, três filhas e de grupos contemporâneos com, no mínimo, três observações. Após essas considerações, a matriz de parentesco ficou com 657 animais no total, e o banco de dado ficou com 1.701 registros de TLN de 421 porcas filhas de 20 cachaços e 120 matrizes, cujo número de observação, média e desvio-padrão referente a cada ordem de parição estão apresentados na Tabela 1.

Tabela 1 - Número de observação (NO), média e desvio-padrão (DP) do tamanho da leitegada por ordem de parição

\begin{tabular}{cccc}
\hline Ordem de parto & NO & Média & DP \\
\hline 1 & 421 & 11,0 & 2,58 \\
2 & 421 & 11,1 & 2,72 \\
3 & 297 & 11,9 & 2,68 \\
4 & 192 & 12,0 & 2,87 \\
5 & 136 & 12,6 & 2,84 \\
6 & 128 & 12,8 & 2,96 \\
7 & 106 & 12,1 & 2,78 \\
\hline
\end{tabular}

O tamanho da leitegada ao nascer foi, inicialmente, analisado por meio de modelo animal, unicaracterística, de regressão aleatória, cujo modelo geral de análise pode ser representado como segue: 
$\mathrm{Y}_{\mathrm{ij}}=\mathrm{F}_{\mathrm{ij}}+\sum_{\mathrm{k}_{\mathrm{\beta}}=0}^{2} \beta_{\mathrm{jk}} \phi_{\mathrm{jk}}+\sum_{\mathrm{k}_{\alpha}=0}^{2} \alpha_{\mathrm{ik}} \phi_{\mathrm{k}}+\sum_{\mathrm{k}_{\mathrm{c}}=0}^{2} \mathrm{c}_{\mathrm{ik}} \phi_{\mathrm{k}}+\sum_{\mathrm{k}_{\mathrm{p}}=0}^{2} \delta_{\mathrm{ik}} \phi_{\mathrm{k}}+\mathrm{e}_{\mathrm{ij}}$

Em que $\mathrm{Y}_{i j}$ é o tamanho da leitegada na $\mathrm{j}_{\text {ésima }}$ ordem de parição, do $\mathrm{i}_{\text {ésimo }}$ animal; $\mathrm{F}_{\mathrm{ij}}$ é o efeito fixo de grupo de contemporâneos (33 níveis), formado pelo ano de parto, estação de parição e pelo rebanho; $\varnothing_{k}$ é o $\mathrm{k}_{\text {ésimo }}$ polinômio de Legendre da ordem de parição padronizada para o intervalo de $-1 \mathrm{a}+1 ; \beta_{j k}$ são os coeficientes de regressão do tamanho da leitegada ao nascer sobre a ordem de parição para modelar a trajetória média da população; $\alpha_{i k}, c_{i k}$ e $\delta_{i k}$ são os coeficientes de regressão aleatória do efeito genético aditivo-direto, do comum-de-leitegada e do de ambiente permanente respectivamente; $k_{\beta}, k_{\alpha}, k_{c} \mathrm{e}$ $k_{p}$ são as ordens dos polinômios de Legendre; $e_{i j}$ é o efeito aleatório residual.

$\mathrm{Na}$ forma matricial, o modelo anterior, com suas pressuposições, pode ser escrito como segue:

$\mathrm{Y}=\mathrm{Xb}+\mathrm{Za}=\mathrm{Hc}+\mathrm{Wp}+\mathrm{e}$

$$
\mathrm{E}\left[\begin{array}{c}
\mathrm{y} \\
\mathrm{a} \\
\mathrm{c} \\
\mathrm{p} \\
\mathrm{e}
\end{array}\right]=\left[\begin{array}{c}
\mathrm{Xb} \\
0 \\
0 \\
0 \\
0
\end{array}\right] ; \quad \mathrm{e} \begin{aligned}
& \mathrm{V}(\mathrm{c})=\mathrm{K}_{\mathrm{c}} \otimes \mathrm{I}_{\mathrm{N} \mathrm{D}} \\
& \mathrm{V}(\mathrm{p})=\mathrm{K}_{\mathrm{p}} \otimes \mathrm{I}_{\mathrm{N} \mathrm{d}} \\
& \mathrm{V}(\mathrm{e})=\mathrm{R}
\end{aligned}
$$

Em que y é o vetor das $N$ observações, medidas em $N_{d}$ animais; $b$ é o vetor de efeitos fixos que inclui as soluções para o grupo contemporâneo; $a, c$ e $p$ são, respectivamente, os vetores dos coeficientes de regressão aleatória do efeito genético aditivo-direto, do comum-deleitegada e do de ambiente permanente; $e$ é o vetor dos diferentes resíduos; X, Z, H e W são, respectivamente, as matrizes de incidência para os efeitos fixos, aleatórios genético aditivo, comum-de-leitegada e de ambiente permanente. $\mathrm{K}_{\mathrm{a}}, \mathrm{K}_{\mathrm{c}}$ e $\mathrm{K}_{\mathrm{p}}$ são, respectivamente, as matrizes de (co)variância entre os coeficientes de regressão aleatória do efeito genético aditivo, comum-de-leitegada e do de ambiente permanente; A é a matriz dos numeradores dos coeficientes de parentesco aditivo; $\mathrm{I}_{\mathrm{Nd}}$ é a matriz identidade de dimensão $\mathrm{Nd}$, sendo $\mathrm{N}_{\mathrm{d}}$ o número de animal com observações repetidas; $I_{N D}$ é a matriz identidade de dimensão $\mathrm{N}_{\mathrm{D}}$, sendo $\mathrm{N}_{\mathrm{D}}$ o número de leitegada; $\otimes$ é o produto de Kroenecker; $\mathrm{R}$ é uma matriz diagonal de variância residual.

A regressão fixa para a curva média da população e as regressões aleatórias do efeito genético aditivo, do comum-de-leitegada e do de ambiente permanente de animal foram modeladas por meio de polinômios ortogonais de Legendre de ordem quadrática, definidas em análises prévias.

Com o fim de averiguar a melhor modelagem para a variância residual, considerou-se a mesma homogênea inicialmente (modelo 1) e, posteriormente, assumiu-se a heterogeneidade de variância por meio de 1 a 7 classes de variância residual. Assim, assumiram-se duas (da $1^{\text {a }}$ à $3^{\mathrm{a}}$ e da $4^{\mathrm{a}}$ à $7^{\mathrm{a}}$ ordem), três (da $1^{\mathrm{a}}$ à $2^{\mathrm{a}}$, da $3^{\mathrm{a}}$ à $4^{\mathrm{a}}$ e da $5^{\mathrm{a}}$ à $7^{\mathrm{a}}$ ordem), quatro $\left(1^{\mathrm{a}}\right.$, da $2^{\mathrm{a}}$ à $3^{\mathrm{a}}$, da $4^{\mathrm{a}}$ à $5^{\mathrm{a}}$ e da $6^{\mathrm{a}}$ à $7^{\mathrm{a}}$ ordem), cinco ( $\left(1^{\mathrm{a}}, 2^{\mathrm{a}}, 3^{\mathrm{a}}\right.$, da $4^{\mathrm{a}}$ à $5^{\mathrm{a}}$ e da $6^{\mathrm{a}}$ à $7^{\mathrm{a}}$ ordem $)$, seis $\left(1^{\mathrm{a}}, 2^{\mathrm{a}}, 3^{\mathrm{a}}, 4^{\mathrm{a}}, 5^{\mathrm{a}}\right.$ e da $6^{\mathrm{a}}$ à $7^{\mathrm{a}}$ ordem) e sete (cada ordem foi assumida como uma classe) classes de variância residual heterogêneas, porém homogêneas dentro de cada grupo.

O número de parâmetro estimado para cada modelo foi $k(k+1) / 2$ coeficientes de regressão para os efeitos genéticos aditivos, comum-de-leitegada e de ambiente permanente, além das classes da variância residual, em que $k$ é a ordem de ajuste do polinômio ortogonal de Legendre.

A estimativa de herdabilidade para cada ordem de parição $(t)$ foi obtida da seguinte forma:

$\mathrm{h}_{\mathrm{t}_{\mathrm{i}}}^{2}=\frac{\hat{\mathrm{g}}_{\mathrm{t}_{\mathrm{i}}}}{\hat{\mathrm{g}}_{\mathrm{t}_{\mathrm{i}}}+\hat{\mathrm{p}}_{\mathrm{t}_{\mathrm{i}}}+\hat{\sigma}_{\mathrm{e}}^{2}}$

$\mathrm{E}$ as estimativas de correlações genéticas e fenotípicas, entre as medidas nas diferentes ordens, $t_{i}$ e $t_{j}$, são definidas, respectivamente, por:

$$
\begin{gathered}
\mathrm{r}_{\hat{g} t_{i} t_{j}}=\frac{\hat{g}_{t_{i} t_{j}}}{\sqrt{\left(\hat{g}_{t_{i} t_{j}} * \hat{g}_{t_{i} t_{j}}\right)}} \\
r_{\hat{p}_{i} t_{j}}=\frac{\hat{p}_{t_{i} t_{j}}}{\sqrt{\left(\hat{p}_{t_{i} t_{j}} * \hat{p}_{\left.t_{i} t_{j}\right)}\right.}}
\end{gathered}
$$

As estimativas de (co)variância genética e fenotípica para a ordem $t$ foram dadas por:

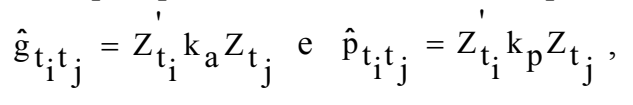

em que $i=1,2,3 \ldots 7$ e $j=1,2,3, \ldots ., 7$.

Os componentes de (co)variância foram estimados pelo método da máxima verossimilhança restrita (REML), usando-se a opção DXMRR do pacote estatístico DFREML (MEYER, 1998).

Os modelos, com diferentes estruturas de variância residual, foram comparados, inicialmente, pela mudança no logaritmo da função de máxima verossimilhança 
(LogL), por meio do teste da razão de verossimilhança (LR). A estatística LR foi obtida pela seguinte expressão: $\mathrm{LR}_{\mathrm{ij}}=2 \log \mathrm{L}_{\mathrm{i}}-2 \log \mathrm{L}_{\mathrm{j}}$, em que $\log \mathrm{L}_{\mathrm{i}}$ é o máximo da função de verossimilhança para o modelo completo $\mathrm{i}$, e $\log \mathrm{L}_{\mathrm{j}}$ é o máximo da função de verossimilhança para o modelo reduzido j. A estimativa LR foi comparada com o valor do qui-quadrado tabelado, com d graus de liberdade e nível de significância de $5 \%$, em que d é a diferença entre o número de parâmetro estimado pelos modelos completo e reduzido.

Também foram utilizados outros testes baseados em formas de máxima verossimilhança restrita, tais como o critério de informação de Akaike (AIC) e o critério de informação bayesiano de Schwarz (BIC). Ambos os testes permitem comparação entre os modelos não aninhados e impõem penalidades de acordo com o número de parâmetro a ser estimado. O modelo com menor valor, para ambos os critérios, é considerado o que obteve melhor ajuste. Segundo Huisman et al. (2002), o BIC é mais rígido que o AIC. Os critérios de informação podem ser definidos como:

$$
\begin{aligned}
& \mathrm{AIC}=-2 \log \mathrm{L}+2 \mathrm{p} \\
& \text { e } \mathrm{BIC}=-2 \log \mathrm{L}+\mathrm{p} \log (\mathrm{N}-\mathrm{r}(\mathrm{X})) .
\end{aligned}
$$

Em que p é o número de parâmetro estimado; N, o número de observação; $r(X)$, o posto da matriz de incidência dos efeitos fixos do modelo; e Log L, o logaritmo da função de máxima verossimilhança restrita (WOLFINGER, 1993).

Posteriormente, o TL foi também analisado por meio de modelo animal de repetibilidade, utilizando-se do aplicativo MTDFREML, descrito por Boldman et al. (1995), em que se considerou, como efeito fixo, o grupo de contemporâneo, como covariável, a ordem de parição e, como aleatórios, os efeitos genético aditivo-direto, ambiental permanente e comum-de-leitegada.
O critério de convergência utilizado foi a variância dos valores do simplex inferior a $10^{-9}$. Após a convergência, o programa era reiniciado, usando-se as estimativas obtidas anteriormente como valores iniciais. Esse procedimento foi repetido até que a diferença entre as estimativas das duas últimas convergências fossem menor que $10^{-9}$.

\section{Resultados e discussão}

Na Tabela 2, estão o número de parâmetro estimado (p), o logaritmo da função de máxima verossimilhança (Log L), critério de informação de Akaike (AIC), critério de informação bayesiano (BIC) e teste da razão de verossimilhança (LRT) para os diferentes MRA.

Analisando-se o Log L, observa-se que, com o aumento das classes de variância de 1 a 7 , houve aumento no $\log \mathrm{L}$, porém essas diferenças não foram significativas ( $P>0,05)$ pelo teste da razão de verossimilhança, o que sugere que o resíduo homogêneo foi suficiente para ajustar a variância nos dados.

Os valores de AIC e BIC também apontaram o modelo 1 como o que proporcionou melhor ajuste da variância nos dados utilizados.

Segundo Meyer (2000), o LRT tende a favorecer modelos com maior número de parâmetro, o que não ocorreu neste trabalho, uma vez que, mesmo aumentando o número de classe residual, não foi observada significância pelo LRT. Já o AIC e o BIC impõem penalidades de acordo com o número de parâmetro a ser estimado; então, à medida que se aumentou o número de classe, como não houve aumento significativo no Log L, não foi observada diminuição no AIC nem no BIC, o que demonstra que o modelo que considerou a

Tabela 2 - Número de parâmetro estimado (Np), logaritmo da função de máxima verossimilhança (Log L), critério de informação de Akaike (AIC), critério de informação bayesiano (BIC) e teste da razão de verossimilhança (LRT) para os diferentes modelos

\begin{tabular}{|c|c|c|c|c|c|}
\hline Modelo & $\mathrm{Np}$ & $\log \mathrm{L}$ & AIC & $\mathrm{BIC}$ & LRT \\
\hline 1 & 19 & $-2588,13$ & 5214,27 & 5318,14 & LRT12 $=0,2^{\text {ns }}$ \\
\hline 2 & 20 & $-2588,03$ & 5216,07 & 5325,41 & LRT23 $=0,04^{\mathrm{ns}}$ \\
\hline 3 & 21 & $-2588,01$ & 5218,03 & 5332,83 & LRT34 $=1,36^{\mathrm{ns}}$ \\
\hline 4 & 22 & $-2587,33$ & 5218,67 & 5338,94 & $\mathrm{LRT} 45=0,66^{\mathrm{ns}}$ \\
\hline 5 & 23 & $-2587,00$ & 5220,00 & 5345,73 & $\operatorname{LRT} 56=0,76^{\mathrm{ns}}$ \\
\hline 6 & 24 & $-2586,62$ & 5221,25 & 5352,45 & LRT67 $=0,16^{\mathrm{ns}}$ \\
\hline 7 & 25 & $-2586,54$ & 5223,09 & 5359,76 & \\
\hline
\end{tabular}
de regressão aleatória

ns = não significativo. Valores em negrito indicam o melhor ajuste com base em cada critério. Modelo 1 - variância residual homogênea; Modelo 2 - duas classes de variância residual; Modelo 3 - três classes de variância residual; Modelo 4 - quatro classes de variância residual; Modelo 5 - cinco classes de variância residual; Modelo 6 - seis classes de variância residual; e Modelo 7 - sete classes de variância residual 
variância residual homogênea (modelo 1) foi o mais indicado para a estrutura dos dados deste trabalho.

Variâncias residuais heterogêneas têm sido relatadas por vários autores, como El Faro et al. (2008) e Sarmento (2007), que trabalharam com bovinos leiteiros e ovinos respectivamente. Essa variação no decorrer de curvas de lactação e de crescimento pode ser atribuída a diversos fatores, como estágio, condições do parto, duração do período seco etc., uma vez que estes não são facilmente incorporados nos modelos de análise devido à falta de escrituração sobre os mesmos. Com isso, essa variação é redirecionada para o resíduo, sendo, portanto, necessário modelá-lo.

O tamanho da leitegada ao nascer sofre influência, principalmente, da covariável ordem de parição, e esse efeito encontra-se ajustado nos modelos aqui utilizados, uma vez que as regressões fixa e aleatórias foram representadas por funções contínuas sobre a ordem de parto. Isso, então, possivelmente explica o fato de o modelo com o resíduo homogêneo ter sido suficiente para modelar a variância nos dados. Associados a esse fato, trabalhos têm utilizado apenas a idade da porca ao parto ou a ordem de parição como covariável na estimativa de parâmetro genético por meio de modelo de repetibilidade para essa característica (IRGANG et al., 1994; TORRES FILHO et al., 2005).

Sabe-se, no entanto, que o tamanho da leitegada é uma característica de baixa herdabilidade, sendo, assim, muito influenciada pelo ambiente. Nesse sentido, não é que a ordem de parição ou a idade de parto da fêmea seja o único fator ambiental que interfere no TLN, mas, sim, que essa covariável talvez seja a única que cause diferenças fenotípicas entre os tamanhos das leitegadas ao longo das ordens de parição, ou seja, outros fatores ambientais, com certeza, afetam o TLN, contudo não são fontes de variação entre as parições, uma vez que devem afetá-las na mesma proporção.

Outros fatores que podem causar diferenças nas ordens de parto são, possivelmente, os efeitos de ano de parto e estação de parição, uma vez que trabalhos mostram influência de tais efeitos (HOLANDA et al., 2005; SILVA et al., 2007). Desse modo, as fêmeas poderiam ter sido cobertas em uma época que, durante a fecundação e a gestação, não era boa devido à falta de disponibilidade de alimento para a produção de ração de boa qualidade, e, por isso, esse tamanho de leitegada ao nascer ter sido prejudicado em relação ao anterior e ao próximo, em virtude da má nutrição. Contudo esses efeitos também estão ajustados através do efeito fixo de grupo de contemporâneo. Caso esses efeitos não estivessem ajustados nos modelos, essa variação iria para o resíduo, tornando-o, possivelmente, diferente (heterogêneo) entre as ordens de parição.
As estimativas de herdabilidade e as proporções de ambiente permanente e de comum-de-leitegada obtidas pelo modelo considerando homogeneidade de variância residual estão apresentadas na Figura 1.

As herdabilidades estimadas pelo MRA que considerou a homogeneidade de variância residual foram próximas a zero, diferindo das obtidas por Lukovic et al. (2004), que, também utilizando modelos de regressão aleatória, observaram herdabilidades variando de 0,09 a 0,14. Mas, corroborando as aqui obtidas, está a de Lourenço (2006), que também obteve herdabilidade próxima de zero (0,001). Holl e Robson (2003), utilizando diferentes métodos de estimação de componentes de variância, encontraram herdabilidades para o tamanho da leitegada ao nascer variando entre 0,00 e 0,16 , o que corrobora as aqui obtidas.

Em relação à estimativa de herdabilidade obtida pelo modelo de repetibilidade, esta foi igual a 0,00 , ou seja, apresentou, praticamente, a mesma magnitude daquelas estimadas por meio do MRA que considerou a homogeneidade de variância residual.

As herdabilidades encontradas na literatura para o tamanho de leitegada, todavia, diferem grandemente de autor para autor. Em geral, a maioria dos autores concorda que o tamanho de leitegada é característica de baixa herdabilidade (CHEN et al., 2003; LUKOVIC et al., 2007; TORRES FILHO et al., 2005).

Em relação à proporção de ambiente permanente (FIG.1), observaram-se diferentes magnitudes em relação às ordens, que foram crescentes da $1^{\text {a }}(0,06)$ à $5^{\text {a }}(0,28)$, e decrescentes desse ponto até a $7^{\mathrm{a}}$ ordem $(0,18)$. Esses resultados são próximos dos relatados por Ferraz e Johnson (1993), porém mais altos que os relatados por Chen et al. (2003) e Lukovic et al. (2004). Pelo modelo de repetibilidade, como a herdabilidade estimada foi igual a 0,00 , o coeficiente

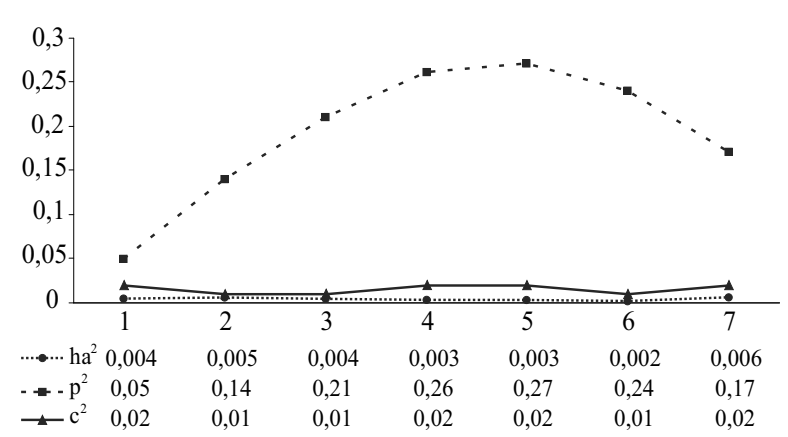

Figura 1 - Herdabilidades $\left(h^{2}{ }_{a}\right)$, proporção do ambiente permanente $\left(p^{2}\right)$ e do comum-de-leitegada $\left(c^{2}\right)$ por ordem de parição de acordo com o modelo que considerou a homogeneidade de variância residual 
de repetibilidade obtido foi igual à proporção do efeito ambiental permanente, ou seja, 0,09 - valor este próximo ao obtido por Lukovic et al. (2007), que foi 0,11.

O efeito comum-de-leitegada, como proporção da variância fenotípica, apresentou, em relação às ordens de parição, valores baixos $(0,01$ a 0,02$)$, corroborando os achados de Lukovic et al. (2004). Por meio do modelo de repetibilidade, a estimativa obtida para esse efeito foi 0,02 .

$\mathrm{Na}$ avaliação genética de suíno, a covariância entre os indivíduos de uma mesma família resulta, além dos genes em comum que esses indivíduos possuem, do ambiente que compartilham durante o período pré-desmame. Esse ambiente comum afeta o desempenho dos animais em toda a sua vida e é determinado, principalmente, pela matriz, através dos seus cuidados com a cria, sua produção de leite etc. (CAVALCANTE-NETO et al., 2009; TORRES FILHO et al., 2005).

Atualmente, em suínos, diversos trabalhos têm utilizado o efeito comum-de-leitegada nos modelos para estimação de componentes de variância, inclusive em características que se manifestam tardiamente na vida da fêmea suína, como as reprodutivas (CAVALCANTE-NETO et al., 2008, 2009; CHIMONYO et al., 2006; KAUFMANN et al., 2000; LUKOVIC et al., 2003, 2004, 2007).

As estimativas de correlação genética direta entre as ordens de parição foram todas positivas e de alta magnitude (TAB. 3). Os valores encontrados na literatura são, na maioria, inferiores aos obtidos neste estudo, como os verificados por Lukovic et al.
(2004), que estimaram correlações entre 0,66 e 0,99. Essa mesma tendência foi relatada por Hanenberg et al. (2001), que encontraram estimativas de correlação genética variando de 0,79 a 0,96 .

As altas correlações genéticas obtidas neste estudo entre as ordens de parição indicam que a maioria dos genes que atuam em uma atua nas demais e no mesmo sentido; disso resulta que os animais melhores nas primeiras parições também o podem ser nas últimas, por exemplo. Entretanto sabe-se que a resposta correlacionada depende não só da correlação genética entre as características mas também das herdabilidades das características envolvidas no processo de seleção. Dessa forma, apesar das altas correlações genéticas entre as ordens de parição, a resposta correlacionada pela seleção indireta, mesmo sendo eficiente, resultará, devido às baixas estimativas de herdabilidade obtidas, em ganhos genéticos muito baixos.

As correlações fenotípicas obtidas foram positivas e variaram de baixa a moderada magnitude (TAB. 3), indicando que os tamanhos de leitegada oriundos de diferentes ordens de parição têm certa dependência fenotípica. A correlação fenotípica entre duas características é composta por dois componentes, um genético e outro de meio. Como as características aqui estudadas $\left(\mathrm{TL}_{1}, \mathrm{TL}_{2}, \ldots, \mathrm{TL}_{7}\right)$ possuem baixas herdabilidades, mesmo com as altas correlações genéticas estimadas entre elas, sabe-se que a correlação fenotípica foi determinada, principalmente, pela contribuição do ambiente. Os resultados obtidos por Lukovic et al. (2004) seguiram essa mesma tendência, ou seja, as correlações

Tabela 3 - Correlações genéticas (abaixo da diagonal) e fenotípicas (acima da diagonal) entre as ordens de parição, estimadas pelo modelo que considerou a homogeneidade de variância residual

\begin{tabular}{cccccccc}
\hline Ordem & 1 & 2 & 3 & 4 & 5 & 6 & 7 \\
\hline 1 & 1 & 0,08 & 0,09 & 0,11 & 0,12 & 0,14 & 0,15 \\
2 & 0,98 & 1 & 0,13 & 0,15 & 0,17 & 0,18 & 0,20 \\
3 & 0,94 & 0,98 & 1 & 0,18 & 0,21 & 0,23 & 0,25 \\
4 & 0,91 & 0,97 & 0,98 & 1 & 0,24 & 0,27 & 0,29 \\
5 & 0,87 & 0,95 & 0,98 & 0,98 & 1 & 0,30 & 0,33 \\
6 & 0,84 & 0,92 & 0,97 & 0,98 & 0,98 & 1 & 0,36 \\
7 & 0,81 & 0,89 & 0,95 & 0,98 & 0,99 & 0,99 & 1 \\
\hline
\end{tabular}


genéticas foram bem superiores às fenotípicas, porém com valores variando de 0,11 a 0,22 .

Estimativas de variância genética aditiva, herdabilidade e correlação genética entre as diferentes parições encontradas por Irgang et al. (1994) para número de leitão nascido vivo e número de leitão aos 21 dias de idade indicaram que a variação genética aditiva disponível para seleção pode ser diferente entre as raças bem como entre as parições intraraça.

\section{Conclusões}

1. A utilização de homogeneidade de variância residual foi mais adequada para modelar as variâncias associadas à característica tamanho da leitegada ao nascer neste conjunto de dado;

2. Os valores de herdabilidade obtidos indicam que, possivelmente, na população estudada, aquela característica não apresentaria ganho genético como resposta à seleção.

\section{Agradecimento}

Às Granjas São José (Monte Alto, São Paulo), especialmente aos irmãos Canalli, e São José (Cabo Verde, Minas Gerais), representada pelo Sr. Adriano Muniz, que, gentilmente, cederam os dados para esta pesquisa.

\section{Referências}

BOLDMAN, K. G. et al. A manual for use of MTDFREML: a set of programs to obtain estimates of variances and covariances. Lincoln: USDA-Agricultural Research Service, 1995. 125 p.

CAVALCANTE-NETO, A. et al. Efeitos genéticos e ambientais sobre a idade à primeira concepção em fêmeas suínas. Arquivo Brasileiro de Medicina Veterinária e Zootecnia, v. 60, n. 02, p. 499-502, 2008.

CAVALCANTE-NETO, A. et al. Estimation models of variance components for farrowing interval in swine. Brazilian Archives Biology and Technology, v. 52, n. 01, p. 69-76, 2009.

CHEN, P. et al. Genetic parameters and trends for litter traits in U.S. Yorkshire, Duroc, Hampshire, and Landrace pigs. Journal of Animal Science, v. 81, n. 01, p. 46-53, 2003.

CHIMONYO, M., DZAMA, K.; BHEBHE, E. Genetic determination of individual birth weight, litter weight and litter size in Mukota pigs. Livestock Science, v. 105, n. 01, p. 69-77, 2006.

EL FARO, L.; ALBUQUERQUE, L. G.; CARDOSO, V. L. Variance component estimates applying random regression models for test-day milk yield in Caracu heifers (Bos taurus Artiodactyla, Bovidae). Genetics and Molecular Biology, v. 31, n. 03, p. 665-673, 2008.

FERRAZ, J. B. S.; JOHNSON, R. K. Animal model estimation of genetic parameters and response to selection for litter size and weight, growth, and backfat in closed seedstock populations of Large White and Landrace swine. Journal of Animal Science, v. 71, n. 04, p. 850-858, 1993.

HANENBERG, E. H. A. T.; KNOL, E. F.; MERKS, J. W. M. Estimates of genetic parameters for reproduction traits at different parities in Dutch Landrace pigs. Livestock Production Science, v. 69, n. 02, p. 179-186, 2001.

HOLANDA, M. C. R. et al. Tamanho da leitegada e pesos médios, ao nascer e aos 21 dias de idade, de leitões da raça Large White. Arquivo Brasileiro de Medicina Veterinária e Zootecnia, v. 57, n. 4, p. 539-544, 2005.

HOLL, J. W.; ROBISON, O. W. Results from nine generations of selection for increased litter size in swine. Journal of Animal Science, v. 81, n. 03, p. 624-629, 2003.

HUISMAN, A. E.; VEERKAMP, R. F.; VAN ARENDONK, J. A. M. Genetic parameters for various random regression models to describe the weight data of pigs. Journal of Animal Science, v. 80, n. 03, p. $575-582,2002$.

IRGANG, R.; FÁVERO, J. A.; KENNEDY, B.Y. Genetic parameters for litter size of diferent parities in Duroc, Landrace and Large White sows. Journal of Animal Science, v. 72, n. 09, p. 2237-2246, 1994.

KAUFMANN, D. et al. Genetic parameters for individual birth and weaning weight for litter size of Large White pigs. Journal of Animal Breeding and Genetics, v. 117, n. 02, p. 121-128, 2000.

LOURENÇO, F. F. Efeitos genéticos sobre a leitegada em suínos puros das raças Landrace e Large White criados no Rio Grande do Sul. 2006. 110f. Dissertação (Mestrado em Zootecnia) - Universidade Federal de Pelotas, Pelotas.

LUKOVIĆ, Z. et al. Genetic parameters for number of piglets born alive using a random regression model. Agriculturae Conspectus Scientificus, v. 68, n. 02, p. 105-108, 2003.

LUKOVIĆ, Z. et al. A random regression model in analysis of litter size in pigs. South African Journal of Animal Science, v. 34, n. 04, p. 241-248, 2004.

LUKOVIĆ, Z.; UREMOVIC, M.; KONJACIC, M. Genetic parameters for litter size in pigs using a random regression model. Asian-australasian journal of animal sciences, v. 20, n. 02, p. 160-165, 2007.

MEYER, K. "DXMRR" A program to estimate covariance functions for longitudinal data by REML. In: WORLD CONGRESS OF GENETICS APPLIED TO LIVESTOCK PRODUCTION, 6., 1998, Armidale, Australia. Proceedings..., Armidale, Australia, 1 CD- Rom.

MEYER, K. Random regression to model phenotypic variation in monthly weights of australian beef cows. Livestock Production Science, v. 65, n. 01, p. 19-38, 2000. 
NOBRE, P. R. C. et al. Analyses of growth curves of Nellore cattle by multiple-trait and random regression models. Journal of Animal Science, v. 81, n. 04, p. 918-926, 2003.

SARMENTO, J. L. R. et al. Avaliação genética de características de crescimento de ovinos Santa Inês utilizando modelos de regressão aleatória. Arquivo Brasileiro de Medicina Veterinária e Zootecnia, v. 58, n. 01, p. 68-77, 2006.

SARMENTO, J. L. R. Modelos de regressão aleatória para avaliação genética da curva de crescimento de ovinos da Raça Santa Inês. 2007. 170f. Tese (Doutorado em Genética e Melhoramento) - Universidade Federal de Viçosa, Viçosa.

SILVA, L. P. G. et al. Influência dos fatores ambientais sobre o tamanho da leitegada ao nascer e taxa de mortalidade à desmama de leitões no brejo paraibano. Ciência Animal Brasileira, v. 08, n. 01, p. 1-6, 2007.

TORRES FILHO, R. A. et al. Estimativas de parâmetros genéticos para características reprodutivas de suínos. Arquivo Brasileiro de Medicina Veterinária e Zootecnia, v. 57, n. 05, p. 684-689, 2005.

VAN DER WERF, J. H. J.; GODDARD, M. E.; MEYER, K. The use of covariance functions and random regressions for genetic evaluation of milk production based on test day records. Journal of Dairy Science, v. 81, n. 12, p. 3300-3308, 1998.

WOLFINGER, R. 1993. Covariance structures selection in general mixed models. Comunications in Statistics, Simulation and Computing, v. 22, n. 04, p. 1079-1106, 1993. 